\title{
Electrical Resistivity of Liquid Metals and Alloys
}

\author{
B. Alkan, R. Karabulut and B. Ünal \\ Department of Engineering Physics, Faculty of Sciences \\ University of Ankara, 06100 Tandoğan, Ankara, Turkey
}

(Received July 31, 2001; revised version April 10, 2002)

\begin{abstract}
New calculations of resistivities of liquid metals, $\mathrm{Na}, \mathrm{K}, \mathrm{Rb}$, and alloys, $\mathrm{Na}-\mathrm{K}, \mathrm{Na}-\mathrm{Rb}, \mathrm{K}-\mathrm{Rb}$, were carried out by using a variant of the Kubo formula that explicitly contains the temperature $T$. The results come closer to the experiment than the previous ones that use the Ziman formula of resistivity.
\end{abstract}

PACS numbers: $72.15 . \mathrm{Cz}$

\section{Introduction}

Ziman formula [1] of resistivity $\rho$ has been, and still continues to be, the most widely used expression in the calculation of liquid metal and binary alloy resistivities. This expression contains a finite integral over the product $Q^{3} a(Q)|\vartheta(Q)|^{2}$ and the upper limit is cut off at $Q=2 k_{\mathrm{f}}$. Here $\vartheta(Q)$ is the pseudopotential due to a single ion, $a(Q)$ is the structure factor and is related to structure amplitude $S(Q)$ via $|S(Q)|^{2}=(\Omega / V) a(Q)$ and $\Omega, V$ are volumes of the unit cell and the system respectively. If we look at it, we can see that it does not contain temperature dependence explicitly. As we know from the experimental curves of structure factor $a(Q)$, temperature dependence comes only from this factor. Therefore, if one wants to obtain a temperature behaviour of the resistivity of any metal, one should use a structure factor $a(Q)$ corresponding to each temperature. This is correct, but it is not adequate. The correct formula thus should involve the temperature $T$ clearly. To achieve this purpose, we have used an expression given by Eq. (1.1) in an earlier paper [2] which is given as Eq. (3.1) in this work. Since the equation is in the form of a ratio, there is a tendency toward balancing the errors coming from the neglect of higher order terms. However, in the Ziman formula there is no 
such a structure, where the errors tend to add up together. At the same time this expression included the temperature dependence through some weight factors in the integrals involved in $\alpha_{r}^{\prime}$ and $\alpha_{r}^{\prime \prime}$ (detailed explanation will be given later or see Ref. [2]). To obtain the temperature behaviour of the resistivity of the pure liquid $\mathrm{Na}$ and $\mathrm{Rb}$, Ashcroft and Lekner [3] have used the structure factors $a(Q)$ which correspond to different temperatures obtained by changing the packing fraction $\eta$ for different pseudopotential $\vartheta(Q)$ in the Ziman formula. We have also used the structure factors obtained from the same packing fraction $\eta$ as theirs in Eq. (3.1) instead of the Ziman formula. It is seen from Fig. 11 that our results are much closer to the experimental values than the other results.

In the calculation of the resistivity of liquid binary alloys as a function of concentration $x$, some physicists have focused on the modification of the pseudopotential so as to obtain satisfactory results compared to the experiment. For this object Ashcroft and Langreth [4] used the volume dependent pseudopotential. On the other hand, Gassert et al. [5] have shown that Fibar-Ziman formalism does not allow the interpretation of the experimental resistivity, if hard-sphere structure factors are used and they suggested that the pseudopotential (and the Ziman formula) must probably be corrected by effective masses. Correction [6, 7] can be made by using

$$
\rho=\left[\left\langle m^{*}\left(k_{\mathrm{f}}\right)\right\rangle\left|N_{k_{\mathrm{f}}}\right|^{2}\right]^{2} \rho_{\mathrm{f}}
$$

where $\rho_{\mathrm{f}}$ stands for the electrical resistivity in the Ziman-type theory. $\left\langle m^{*}\left(k_{\mathrm{f}}\right)\right\rangle$ is the density-of-states effective mass averaged over the Fermi surface and $N_{k_{\mathrm{f}}}$ is the normalization constant of the state $\boldsymbol{k}$. The values of the calculated resistivity with the Ziman formula as a function of concentration $x$ generally are below the experimental curves. Therefore, by using the above equation one achieves a close curve to the experimental one, because the pre-multiplier $\left\langle m^{*}\left(k_{\mathrm{f}}\right)\right\rangle$ is bigger than unity, although not very much. This is seen from Table III given in Ref. [6]. Although in this work we did not use such a correction, we have obtained a curve close to the experiment. A detailed argument will be given in the next section.

Besides the mentioned conventional techniques recently some modern techniques such as computer simulation, direct evaluation of conductivity formula by ab initio molecular dynamics simulation have been developed [8-10]. However, although it has been possible to come very close to the experiment in some property calculations, these new techniques have not reached yet to a satisfactory level in transport property calculations. Our purpose has been to show that the Kubo formula gives better results than the simple Ziman equation of resistivity under the same assumptions.

In Secs. 2 and 3 we introduce the mathematical model of the problem and formulation of resistivity respectively. In Sec. 4 the correlation functions $\alpha_{r}^{\prime}$ and $\alpha_{r}^{\prime \prime}$ are calculated. Sections 5 and 6 contain the calculation of resistivities of liquid pure metals and alloys, and we discuss the results in the final section. 


\section{Pseudopotential model for liquid metals and alloys}

In liquid metals the coupling between the carriers and the rest part of many-particle system is given by the local pseudopotential $U[11,12]$

$$
U=\sum_{\boldsymbol{k}, q} S(q) \vartheta(q) a_{k+q}^{+} a_{k},
$$

where $S(q)$ and $\vartheta(q)$ are structure amplitude and form factor, $a_{k+q}^{+}$and $a_{k}$ are creation and annihilation operators of carriers with wave vectors $\boldsymbol{k}+\boldsymbol{q}$ and $\boldsymbol{k}$, respectively.

Equation (2.1) can be extended to binary alloys [13] by replacing the square of the absolute value of $S(q) \vartheta(q)$ involved in it by

$$
\sqrt{x a_{22}(q) \vartheta_{2}^{2}(q)+2[x(1-x)]^{1 / 2} a_{12}(q) \vartheta_{2}(q) \vartheta_{1}(q)+(1-x) a_{11}(q) \vartheta_{1}^{2}(q)} .
$$

Here $\vartheta_{i}(q)$ are the interactions of an electron with ions of species $i(i=1,2)$ immersed in the same screening cloud of electrons. $x$ is the concentration of species 2. $a_{i j}(q)$ are partial structure factors describing a mixture of randomly distributed hard spheres with different diameters, which is the hard-sphere momentum-space solutions of the Percus-Yevick equation for the radial distribution function in a classical fluid [14]. The $a(q)$ derived in this way gave very good agreement with the X-ray and neutron-scattering data around the first peak. A detailed information about it then will be given in the text. Werner and Fresard [15] in their work on temperature dependence of transport coefficients in liquid and amorphous metals have also used the same model. In order to facilitate direct comparison of our results with the Ziman expression based calculations we preferred the hard-sphere model.

\section{Formulation of resistivity for liquid metals and binary alloys}

As we mentioned above, because the Ziman expression is insufficient, we need an alternative formula containing all these important effects. For this we can rely on the well known Kubo formula. In this work we used the following expression [2] based on the Kubo formula for the calculation of the resistivity of pure liquid metals and alloys

$$
\rho=\frac{6 V\left(\alpha_{r}^{\prime}\right)^{2}}{e^{2} N^{2} \alpha_{r}^{\prime \prime}}
$$

where $\alpha_{r}^{\prime}$ and $\alpha_{r}^{\prime \prime}$ are first and second derivatives with respect to frequency $\omega$, of real parts of force-force correlation function [16] $\alpha(\omega)$ (formula (2.18) in Ref. [16]). Other symbols have their customary meaning. For a pure liquid metal $\alpha_{r}^{\prime}$ and $\alpha_{r}^{\prime \prime}$ are given by [2]

$$
\alpha_{r}^{\prime}=\frac{2 \pi}{3} \sum_{\boldsymbol{k}, q} \hbar^{2} q^{2}|S(q)|^{2} \vartheta^{2}(q) n_{k}^{\prime} \delta^{\prime}\left(\Delta E^{\prime}\right),
$$




$$
\alpha_{r}^{\prime \prime}=-\frac{2 \pi}{3} \sum_{\boldsymbol{k}, q} \hbar^{3} q^{2}|S(q)|^{2} \vartheta^{2}(q) n_{k}^{\prime} \delta^{\prime \prime}\left(\Delta E^{\prime}\right) .
$$

If we use Eq. (2.2) in Eqs. (3.2), (3.2a), for $\alpha_{r}^{\prime}$ and $\alpha_{r}^{\prime \prime}$ connected with binary alloys we have

$$
\begin{aligned}
\alpha_{r}^{\prime}= & \frac{2 \pi}{3} \sum_{\boldsymbol{k}, q} \hbar^{2} q^{2}\left\{x\left|S_{22}(q)\right|^{2} \vartheta_{2}^{2}(q) n_{k}^{\prime} \delta^{\prime}\left(\Delta E^{\prime}\right)\right. \\
& +2[x(1-x)]^{1 / 2}\left|S_{12}(q)\right|^{2} \vartheta_{1}(q) \vartheta_{2}(q) n_{k}^{\prime} \delta^{\prime}\left(\Delta E^{\prime}\right) \\
& \left.+(1-x)\left|S_{11}(q)\right|^{2} \vartheta_{1}^{2}(q) n_{k}^{\prime} \delta^{\prime}\left(\Delta E^{\prime}\right)\right\} \\
\alpha_{r}^{\prime \prime}= & -\frac{2 \pi}{3} \sum_{k, q} \hbar^{3} q^{2}\left\{x\left|S_{22}(q)\right|^{2} \vartheta_{2}^{2}(q) n_{k}^{\prime} \delta^{\prime \prime}\left(\Delta E^{\prime}\right)\right. \\
& +2[x(1-x)]^{1 / 2}\left|S_{12}(q)\right|^{2} \vartheta_{1}(q) \vartheta_{2}(q) n_{k}^{\prime} \delta^{\prime \prime}\left(\Delta E^{\prime}\right) \\
& \left.+(1-x)\left|S_{11}(q)\right|^{2} \vartheta_{1}^{2}(q) n_{k}^{\prime} \delta^{\prime \prime}\left(\Delta E^{\prime}\right)\right\} .
\end{aligned}
$$

We shall use these expressions in the resistivity formula (3.1). The sums in the formulae (3.2), (3.2a), (3.3), and (3.3a) will be carried out in the following section.

\section{Evaluation of $\alpha_{r}^{\prime}$ and $\alpha_{r}^{\prime \prime}$}

In order to calculate the resistivity of the pure liquid metals from Eq. (3.2), we should do the sums in Eqs. (3.2), (3.2a). These sums may be carried out by turning them into integrations in the usual way by using the relations

$$
\sum_{q} \rightarrow \frac{V}{(2 \pi)^{3}} \int \mathrm{d}^{3} q, \quad \sum_{k} \rightarrow \frac{V}{(2 \pi)^{3}} \int \mathrm{d}^{3} k .
$$

During the calculation of the resistivity, we assume that the energy spectra of the considered system is parabolic

$$
E(k)=\frac{\hbar^{2} k^{2}}{2 m} \text {. }
$$

If we denote the angle between the wectors $k$ and $q$ by $\theta$ and call $\cos \theta=\nu$, the energy difference $\Delta E^{\prime}$ becomes

$$
\Delta E^{\prime}=\frac{\hbar^{2} k q}{m} \nu+\frac{\hbar^{2} q^{2}}{2 m}
$$

to obtain the condition $\Delta E^{\prime}=0$, the variable $\nu$ must be restricted to negative region $-1 \leq \nu \leq 0$. Integration is carried out in the order $\mathrm{d} \nu \rightarrow \mathrm{d}(\hbar q) \rightarrow \mathrm{d}(\hbar k)$ because with this order, during the transformation of delta functions into simpler forms Jacobians never become zero, otherwise nothing fixes this order. A detailed 
information about the calculation of $\alpha_{r}^{\prime}$ and $\alpha_{r}^{\prime \prime}$ are given in Appendix and the results are

$$
\begin{aligned}
\alpha_{r}^{\prime}= & \frac{V \beta m^{2} k_{\mathrm{f}}^{4} \Omega}{24 \pi^{3} \hbar^{2}} I_{1}, \\
I_{1}= & \int_{0}^{\infty} \mathrm{d} Q Q^{3} a(Q) \vartheta^{2}(Q) \frac{\exp \left[\beta E_{\mathrm{f}}\left(\frac{Q^{2}}{4}-1\right)\right]}{\left\{\exp \left[\beta E_{\mathrm{f}}\left(\frac{Q^{2}}{4}-1\right)\right]+1\right\}^{2}}, \\
\alpha_{r}^{\prime \prime}= & \frac{V \beta^{2} m^{2} k_{\mathrm{f}}^{4} \Omega}{24 \pi^{3} \hbar} I_{2}, \\
I_{2}= & \frac{1}{\beta E_{\mathrm{f}}} \int_{0}^{\infty} \mathrm{d} Q Q a(Q) \vartheta^{2}(Q) \frac{\exp \left[\beta E_{\mathrm{f}}\left(\frac{Q^{2}}{4}-1\right)\right]}{\left\{\exp \left[\beta E_{\mathrm{f}}\left(\frac{Q^{2}}{4}-1\right)\right]+1\right\}^{2}} \\
& +\frac{2}{\beta E_{\mathrm{f}}} \int_{0}^{\infty} \mathrm{d} Q Q^{2} a(Q) \vartheta(Q) \vartheta^{\prime}(Q) \frac{\exp \left[\beta E_{\mathrm{f}}\left(\frac{Q^{2}}{4}-1\right)\right]}{\left\{\exp \left[\beta E_{\mathrm{f}}\left(\frac{Q^{2}}{4}-1\right)\right]+1\right\}^{2}} .
\end{aligned}
$$

On the same basis for the binary alloys $\alpha_{r}^{\prime}$ and $\alpha_{r}^{\prime \prime}$ are given by

$$
\begin{aligned}
& \alpha_{r}^{\prime}= \frac{V \beta m^{2} k_{\mathrm{f}}^{4} \Omega}{24 \pi^{3} \hbar^{2}} I_{1}, \\
& I_{1}= x \int_{0}^{\infty} \mathrm{d} Q Q^{3} a_{2}(Q) \vartheta^{2}(Q) \frac{\exp \left[\beta E_{\mathrm{f}}\left(\frac{Q^{2}}{4}-1\right)\right]}{\left\{\exp \left[\beta E_{\mathrm{f}}\left(\frac{Q^{2}}{4}-1\right)\right]+1\right\}^{2}} \\
&+2[x(1-x)]^{1 / 2} \int_{0}^{\infty} \mathrm{d} Q Q^{3} a_{12}(Q) \vartheta_{1}(Q) \vartheta_{2}(Q) \frac{\exp \left[\beta E_{\mathrm{f}}\left(\frac{Q^{2}}{4}-1\right)\right]}{\left\{\exp \left[\beta E_{\mathrm{f}}\left(\frac{Q^{2}}{4}-1\right)\right]+1\right\}^{2}} \\
&+(1-x) \int_{0}^{\infty} \mathrm{d} Q Q^{3} a_{1}(Q) \vartheta_{1}^{2}(Q) \frac{\exp \left[\beta E_{\mathrm{f}}\left(\frac{Q^{2}}{4}-1\right)\right]}{\left\{\exp \left[\beta E_{\mathrm{f}}\left(\frac{Q^{2}}{4}-1\right)\right]+1\right\}^{2}}, \\
& \alpha_{r}^{\prime \prime}= \frac{V \beta^{2} m^{2} k_{\mathrm{f}}^{4} \Omega}{24 \pi^{3} \hbar} I_{2},
\end{aligned}
$$




$$
\begin{aligned}
I_{2}= & \frac{x}{\beta E_{\mathrm{f}}}\left\{\int_{0}^{\infty} \mathrm{d} Q Q a_{2}(Q) \vartheta_{2}^{2}(Q) \frac{\exp \left[\beta E_{\mathrm{f}}\left(\frac{Q^{2}}{4}-1\right)\right]}{\left\{\exp \left[\beta E_{\mathrm{f}}\left(\frac{Q^{2}}{4}-1\right)\right]+1\right\}^{2}}\right\} \\
& \left.+2 \int_{0}^{\infty} \mathrm{d} Q Q^{2} a_{2}(Q) \vartheta_{2}(Q) \vartheta_{2}^{\prime}(Q) \frac{\exp \left[\beta E_{\mathrm{f}}\left(\frac{Q^{2}}{4}-1\right)\right]}{\left\{\exp \left[\beta E_{\mathrm{f}}\left(\frac{Q^{2}}{4}-1\right)\right]+1\right\}^{2}}\right\} \\
& \left.+\beta E_{\mathrm{f}}\right]^{1 / 2}\left\{\int_{0}^{\infty} \mathrm{d} Q Q a_{12}(Q) \vartheta_{1}(Q) \vartheta_{2}(Q) \frac{\exp \left[\beta E_{\mathrm{f}}\left(\frac{Q^{2}}{4}-1\right)\right]}{\left\{\exp \left[\beta E_{\mathrm{f}}\left(\frac{Q^{2}}{4}-1\right)\right]+1\right\}^{2}}\right. \\
& +\int_{0}^{\infty} \mathrm{d} Q Q^{2} a_{12}(Q) \vartheta_{1}(Q) \vartheta_{2}^{\prime}(Q) \frac{\exp \left[\beta E_{\mathrm{f}}\left(\frac{Q^{2}}{4}-1\right)\right]}{\left\{\exp \left[\beta E_{\mathrm{f}}\left(\frac{Q^{2}}{4}-1\right)\right]+1\right\}^{2}} \\
& \left.+\int_{0}^{\infty} \mathrm{d} Q Q^{2} a_{12}(Q) \vartheta_{1}^{\prime}(Q) \vartheta_{2}(Q) \frac{\exp \left[\beta E_{\mathrm{f}}\left(\frac{Q^{2}}{4}-1\right)\right]}{\left\{\exp \left[\beta E_{\mathrm{f}}\left(\frac{Q^{2}}{4}-1\right)\right]+1\right\}^{2}}\right\} \\
& +\frac{(1-x)}{\beta E_{\mathrm{f}}}\left\{\int_{0}^{\infty} \mathrm{d} Q Q Q^{2} a_{1}(Q) \vartheta_{1}(Q) \vartheta_{1}^{\prime}(Q) \frac{\exp \left[\beta E_{\mathrm{f}}\left(\frac{Q^{2}}{4}-1\right)\right]}{\left.\left\{\exp \left[\beta E_{\mathrm{f}}\left(\frac{Q^{2}}{4}-1\right)\right]+1\right\}^{2}\right\}}(4.7 \mathrm{a})\right. \\
& +\exp \left[\beta E_{\mathrm{f}}\left(\frac{Q^{2}}{4}-1\right)\right] \\
& \left.\left.\left.+\frac{Q^{2}}{4}-1\right)\right]+1\right\}^{2}
\end{aligned}
$$

We shall use these expressions in the resistivity formula (3.1).

\section{Electrical resistivity of liquid metals}

In this section we have applied the resistivity formula (3.1) to the pure liquid metals $\mathrm{Na}, \mathrm{Rb}, \mathrm{K}$ in order to see the temperature behaviour of them. To obtain this object, we should calculate the integrals $I_{1}, I_{2}$ given by (4.4a) and (4.5a) and which are involved in $\alpha_{r}^{\prime}$ and $\alpha_{r}^{\prime \prime}$ of the resistivity formula (3.1). Since integrals $I_{1}, I_{2}$ both contain $a(Q)$ and $\vartheta(Q)$, the values of these integrals depend on these sensitively. During the calculation of resistivities of three different liquid metals $\mathrm{Na}, \mathrm{K}, \mathrm{Rb}$ we use the theoretical curve of $a(Q)$, which is the solution of the Percus-Yevick equation. The curve of $a(Q)$ so obtained depends only on one parameter, a general packing fraction $\eta$, which is related with hard-sphere diameter $\sigma$ by $\eta=(\pi / 6) n \sigma^{3}$, and $n$ is the free carrier concentration. The form factor $\vartheta(Q)$ of the pseudo-atom is supposed to be weaker and much more rapidly convergent than the Fourier transform of the true atomic potential, but which yields essentially the same band structure. 
In obtaining the resistivity of liquid $\mathrm{Na}$, we have used the form factor $\vartheta(Q)$ of the model potential of Heine and Abarenkov obtained by Animalu and Heine whose data were tabulated as table 8.4 in Harrison's book [12] and in order to see the $Q$ dependence clearly we drew Fig. 1 using these data, and for the structure factor we have used a theoretical curve obtained from the solution of the Percus-Yevick equation as given in Fig. 2. The latter contains some curves corresponding to different packing fractions $\eta$ which are taken from the Ashcroft et al. work [3]. In literature $T$ dependence of $\eta$ is often determined through using Hasegawa formula [17], however since we wanted to compare our results with those of Ref. [3], we have used their $\eta$ values. Temperature dependence of $\eta$ can also be seen from Fig. 3.6 of Waseda's book [18]. For example we read $\eta=0.462$ at $T=100^{\circ} \mathrm{C}$ and $\eta=0.414$ at $T=300^{\circ} \mathrm{C}$ from his figure for liquid $\mathrm{Na}$. We calculated the resistivities as $\rho=9.05 \mu \Omega \mathrm{cm}$ and $\rho=14.32 \mu \Omega \mathrm{cm}$ corresponding to the above $\eta$ values respectively. Similarly for liquid $\mathrm{K}$ we read $\eta=0.462$ at $T=65^{\circ} \mathrm{C}$ and $\eta=0.423$ at $T=195^{\circ} \mathrm{C}$ and calculated resistivities are $\rho=12.43 \mu \Omega \mathrm{cm}$ and $\rho=20.99 \mu \Omega \mathrm{cm}$ respectively. These results do not differ very much from the ones in Table I and in Table III which shall be presented shortly.

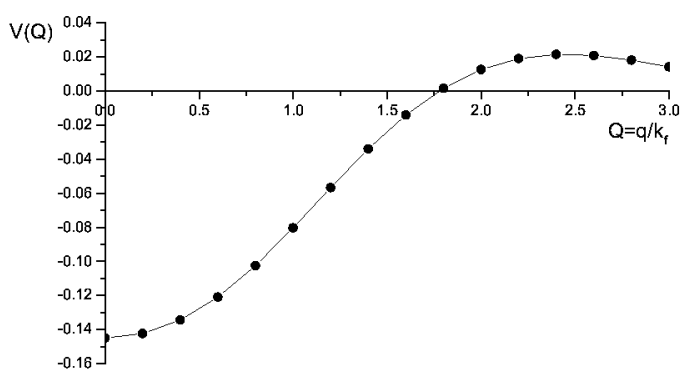

Fig. 1. Form factor of the model potential of Heine and Abarenkov for sodium as function of the momentum transfer $q$ (drawing based on the data from [12]).

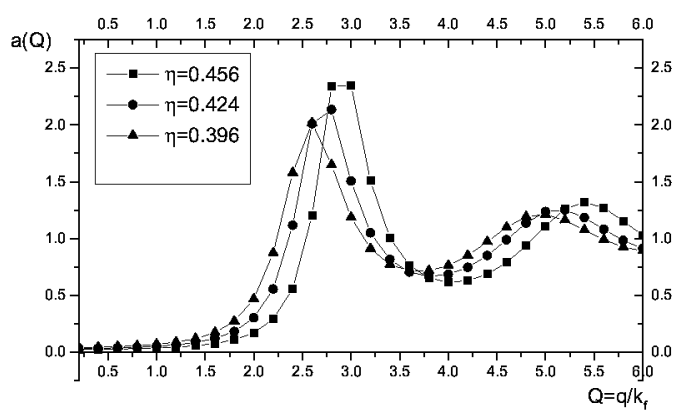

Fig. 2. Structure factor $a(Q)$ of a fluid of hard spheres according to the Percus-Yevick equation for sodium for different packing fraction $\eta$. 
TABLE I

Temperature dependence of the resistivity of liquid sodium. The values of the resistivities in $\mu \Omega \mathrm{cm}$ unit. The third column is our resistivity values and the fourth is the experiment. The other columns show the resistivities given in Ref. [3] for different form factors.

\begin{tabular}{c|c|c|c|c|c|c}
\hline \hline$T\left[{ }^{\circ} \mathrm{C}\right]$ & $\eta=(\pi / 6) n \sigma^{3}$ & $\rho[\mu \Omega \mathrm{cm}]$ & $\rho_{\exp }$ & $\rho_{\mathrm{C}}$ & $\rho_{\mathrm{HA}}$ & $\rho_{\mathrm{HAA}}$ \\
\hline 100 & 0.456 & 9.51 & 9.6 & 7.6 & 9 & 5 \\
160 & 0.437 & 11.67 & 11.7 & 9.1 & 10.7 & 5.9 \\
200 & 0.424 & 12.99 & 13.1 & 10.3 & 12.1 & 6.6 \\
240 & 0.423 & 14.39 & 14.5 & 11.4 & 13.3 & 7.2 \\
300 & 0.396 & 16.48 & 16.6 & 13.6 & 15.4 & 8.3
\end{tabular}

TABLE II

Temperature dependence of the resistivity of liquid $\mathrm{Rb}$. The values of the resistivities in $\mu \Omega \mathrm{cm}$ unit. The third column is our resistivity values and the fourth is the experiment. The other columns show the resistivities given in Ref. [3] for different form factors.

\begin{tabular}{c|c|c|c|c|c|c}
\hline \hline$T\left[{ }^{\circ} \mathrm{C}\right]$ & $\eta=(\pi / 6) n \sigma^{3}$ & $\rho[\mu \Omega \mathrm{cm}]$ & $\rho_{\exp }$ & $\rho_{\mathrm{c}}$ & $\rho_{\mathrm{HA}}$ & $\rho_{\mathrm{HAA}}$ \\
\hline 40 & 0.459 & 21.32 & 22 & 8.7 & 25.4 & 7.5 \\
160 & 0.412 & 32.38 & 33 & 13.9 & 36.1 & 11.7 \\
240 & 0.395 & 39.69 & 40.5 & 16.8 & 41.1 & 13.8 \\
360 & 0.365 & 51.22 & 52 & 22.8 & 50.9 & 18.1
\end{tabular}

TABLE III

Temperature dependence of the resistivity of liquid potassium. The values of the resistivities in $\mu \Omega \mathrm{cm}$ unit.

\begin{tabular}{c|c|c|c}
\hline \hline$T\left[{ }^{\circ} \mathrm{C}\right]$ & $\eta=(\pi / 6) n \sigma^{3}$ & $\rho[\mu \Omega \mathrm{cm}]$ & $\rho_{\exp }$ \\
\hline 65 & 0.443 & 12.6 & 13 \\
130 & 0.438 & 15.6 & 16.7 \\
195 & 0.433 & 20 & 20.3 \\
260 & 0.428 & 24 & 24.5
\end{tabular}

All the numerical results so obtained for liquid $\mathrm{Na}$ are given in Table $\mathrm{I}$. Values of $\Omega$ and $E_{\mathrm{f}}$ required for these calculations have been taken as $39.3 \times$ $10^{-30} \mathrm{~m}^{3}, 0.30 \times 10^{-18} \mathrm{~J}$, respectively [2]. 
In the similar way, for $\mathrm{Rb}, \mathrm{K}$ we use the values of $\vartheta(Q)$ and $a(Q)$ given by Fig. 3, Fig. 4, Fig. 5 and Fig. 6 respectively. Numerical results are listed in Table II and Table III.

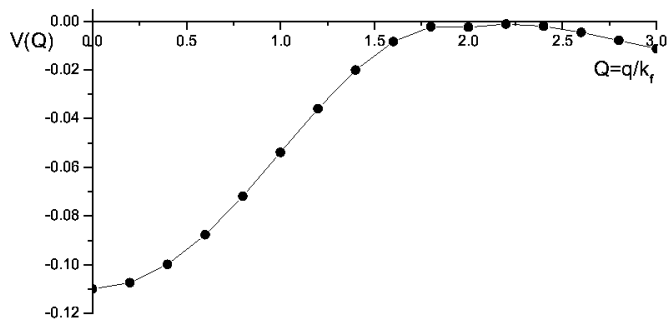

Fig. 3. Form factor of the model potential of Heine and Abarenkov for Rb as function of the momentum transfer $q$ (drawing based on the data from [12]).

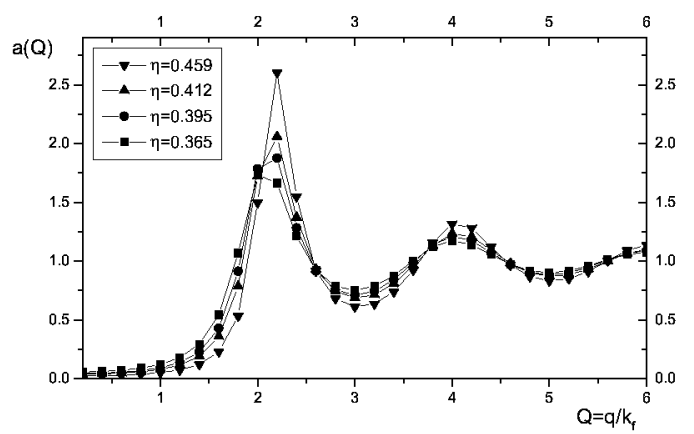

Fig. 4. Structure factor $a(Q)$ of a fluid of hard spheres according to the Percus-Yevick equation for Rb for different packing fraction $\eta$.

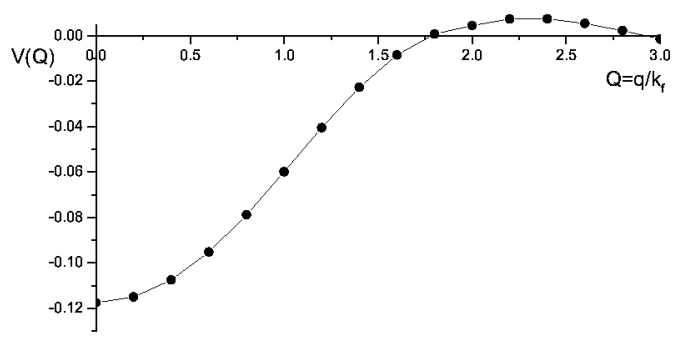

Fig. 5. Form factor of the model potential of Heine and Abarenkov for $\mathrm{K}$ as function of the momentum transfer $q$ (drawing based on the data from [12]).

For $\mathrm{Rb}$, values of $\Omega$ and $E_{\mathrm{f}}$ required for these calculations have been taken as $9.54 \times 10^{-29} \mathrm{~m}^{3}, 0.29 \times 10^{-18} \mathrm{~J}$, respectively $[7,12]$.

In the calculation of the resistivity of the $\mathrm{K}$ we used the $79.5 \times 10^{-30} \mathrm{~m}^{3}$, $0.327 \times 10^{-18} \mathbf{J}$ for $\Omega, E_{\mathrm{f}}$, respectively $[7,12]$. 


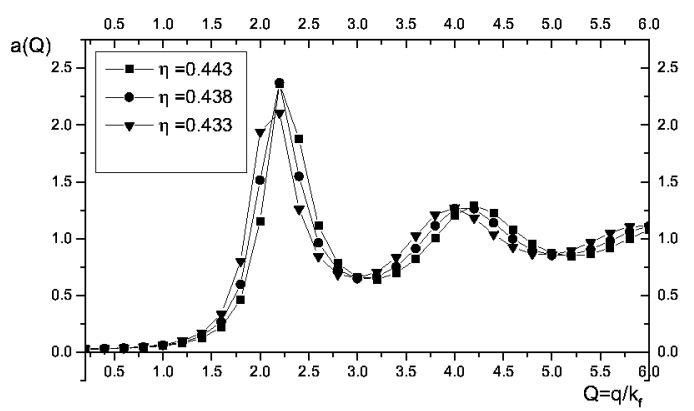

Fig. 6. Structure factor $a(Q)$ of a fluid of hard spheres according to the Percus-Yevick equation for $\mathrm{K}$ for different packing fraction $\eta$.

For the liquid $\mathrm{K}$, we have determined the parameter $\eta$ by fitting $a(Q)$ to give the correct height, $a_{\max }$, of the structure factor as described in Ref. [3].

If we look at Tables I, II, III, it is seen that for these liquid metals, Na, $\mathrm{Rb}, \mathrm{K}$, each value of the resistivity corresponding to the different temperatures $T$ we obtained is closer to the experimental one than the values obtained by other means. The detailed argument connected with these results will be given in the conclusions.

\section{Electrical resistivities of liquid alloys}

In obtaining the resistivity of liquid binary alloys, $\mathrm{Na}-\mathrm{K}, \mathrm{Na}-\mathrm{Rb}, \mathrm{K}-\mathrm{Rb}$, we use Eqs. (4.6), (4.7) involved in the resistivity formula (3.1) in place of Eqs. (4.4), (4.5). During the evaluation of these expressions we use again the same form factor $\vartheta(Q)$ for each component of the alloys, but partial structure factor have been considered. Some of the partial structure factors calculated, to be used in the calculation of the resistivity in this paper, using the solution of the Percus-Yevick equation and denoted by $a_{i j}(Q)$ are shown as examples in Fig. 7 .

The variables $\eta, \alpha$, and $x$ of the partial structure factor specify the system. $\eta$ is the total packing fraction for the mixture. Although the Percus-Yevick equation shows no singularities for $\eta<1$, the region beyond $\eta=0.74$ is nonphysical, since the fluid then has a packing density greater than that of the close-packed solid. $\alpha$ is defined to be the hard-sphere ratio of the alloys. It takes the values between 0 and 1. $x$ is defined being the concentration of larger spheres.

A plot of resistivity for the $\mathrm{Na}-\mathrm{K}$ alloys against $x$ is given in Fig. 8. The experimental data are taken from Hannephof et al. [19].

In Figs. 9, 10 we show the experimental and theoretical resistivity curves which are plotted versus $x$ for $\mathrm{Na}-\mathrm{Rb}, \mathrm{K}-\mathrm{Rb}$ liquid alloys.

When we look at Figs. 8, 9, 10 we conclude that the concentration behaviour of the resistivity of liquid binary alloys can be roughly described by Nordheim-type parabolas as expected, and they are very close to experimental curves. A detailed information will be given in the conclusions. 



Fig. 7. The partial structure factors presently calculated and used for (a) the $\mathrm{Na}-\mathrm{K}$ alloys at $x_{\mathrm{K}}=0.2$ and $T=100^{\circ} \mathrm{C}$, (b) the Na-Rb alloys at $x_{\mathrm{Rb}}=0.2$ and $T=100^{\circ} \mathrm{C}$, (c) the $\mathrm{K}-\mathrm{Rb}$ alloys at $x_{\mathrm{Rb}}=0.2$ and $T=100^{\circ} \mathrm{C}$.

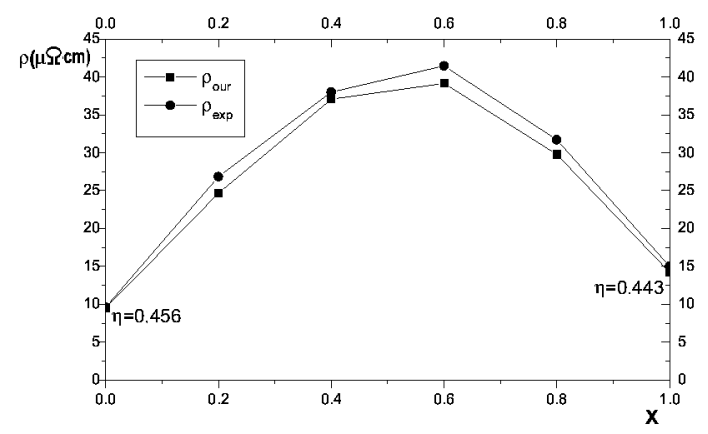

Fig. 8. Electrical resistivity calculated using Eq. (3.1) for $\mathrm{Na}-\mathrm{K}$ for $\alpha=0.8$ at $100^{\circ} \mathrm{C}$, denoted by (- - ). The corresponding experimental results are represented by $(-\bullet-)$. 




Fig. 9. Electrical resistivity calculated using Eq. (3.1) for $\mathrm{Na}-\mathrm{Rb}$ for $\alpha=0.9$ at $100^{\circ} \mathrm{C}$, denoted by $(-\bullet-)$. Corresponding experimental results are represented by (- -$)$.

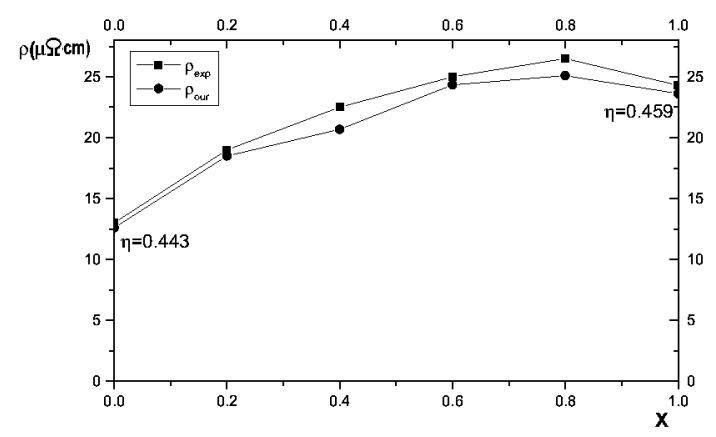

Fig. 10. Electrical resistivity calculated using Eq. (3.1) for $\mathrm{K}-\mathrm{Rb}$ for $\alpha=0.7$ at $65^{\circ} \mathrm{C}$,

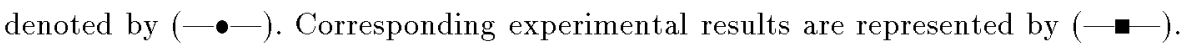

\section{Conclusions}

Our results for liquid $\mathrm{Na}$ are given in Table I and Fig. 11a. In obtaining these we used the same pseudopotential and structure factor as used in Ref. [3], but the resistivity formula Eq. (3.1) we used was different. As is seen from Fig. 11a our resistivities are much closer to the experiment than Ashcroft and Lekner [3]. Table II and Fig. 11b show the results of our calculations for Rb, these values are also closer to the experiment than the results of Ref. [3]. Table III and Fig. 11c are about the resistivities of $\mathrm{K}$, which are close to the experiment. There is a real improvement in all the results which comes from the explicit $T$ dependence of the resistivity formula $\mathrm{Eq}$. (3.1) based on the Kubo formula.

The results for $\mathrm{Na}-\mathrm{K}$ alloy are shown in Figs. 7a, 8. It is seen from Fig. 8 that our resistivity values are closer to the experiment than all the results of Refs. [4, 6] and [7]. Besides Ref. [3] uses volume dependent pseudopotential and we use the standard one which is widely known in the literature. Since the resistivity depends sensitively on the choice of pseudopotential we avoided any changes on it. On the other hand, in Refs. [6] and [7] they use a correction factor to the Ziman equation, 

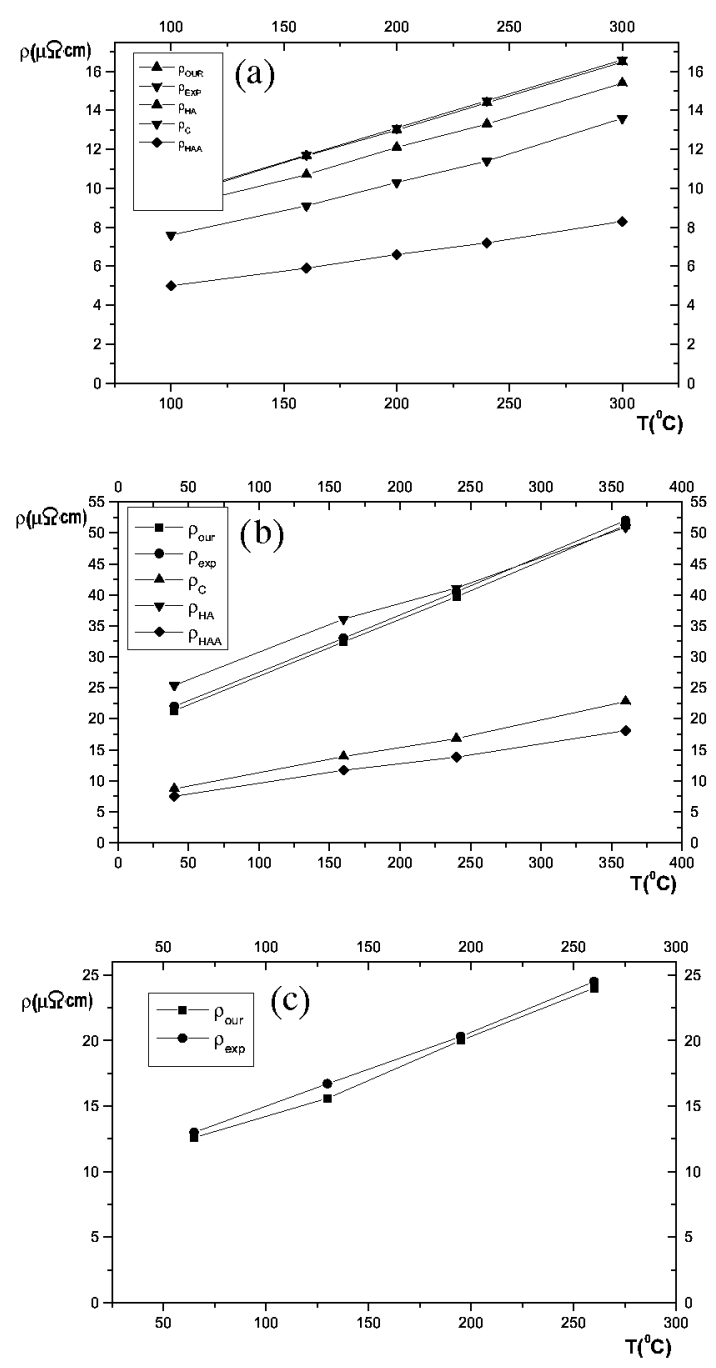

Fig. 11. Temperature behaviour of the resistivity of the pure liquid metal (a) for Na, (b) for $\mathrm{Rb},(\mathrm{c})$ for $\mathrm{K}$.

given by Eq. (1.1). By this they improved their results greatly, but since our results are already close enough to the experiment, we did not force ourselves to use such a factor.

For $\mathrm{Na}-\mathrm{Rb}$ and $\mathrm{K}-\mathrm{Rb}$ alloys we give the results in Figs. $7 \mathrm{~b}, 9$ and $7 \mathrm{c}, 10$ respectively. Again resistivity versus concentration curves are satisfactorily close to the experiment and similar arguments as said for the $\mathrm{Na}-\mathrm{K}$ case can again be repeated here.

There are recently developed modern techniques based on computer simulation. We are aware of this fact, but we saw that these techniques are, at the 
moment, very far from being satisfactory. For example, if we look at the Silvestrelli et al. work [9] on electrical conductivity calculation in ab initio simulations of metals application to liquid sodium, their Fig. 10 shows that calculated conductivities do not agree with the experiment. This fact is expressed in their words as "we find that, even using a proper $k$-point sampling, our results are in agreement with the experimental data for temperature higher than $700 \mathrm{~K}$ only". This shows that this kind of numerical work, although very attractive, has not reached to satisfactory level even for the simplest liquid alkali metal sodium.

Another example of a modern study is the Kirchhoff et al. work [8] about structure, dynamics, and electronic structure of liquid $\mathrm{Ag}-\mathrm{Se}$ alloys investigated by ab initio simulation. Calculated conductivity results are given in their Table $\mathrm{V}$ at different material compositions $x$. In their words they say that "as $x$ increases, conductivity first increases dramatically to $\approx 1500 \Omega^{-1} \mathrm{~cm}^{-1}$ at $x=0.42$ and then decreases slightly to $\approx 1250 \Omega^{-1} \mathrm{~cm}^{-1}$ at $x=0.65$. These values are $3-4$ times higher than the experimental values". They dare to attribute this discrepancy to the Kubo formula itself by saying "we believe that these large discrepancies with experiment arise from the approximations implicit in the Kubo-Greenwood approach". We do not carry their view, because the Kubo formula is universally accepted, so it is better to question their technique. We can give maybe other examples of work that are similar to the above mentioned ones, for example Kaschner et al. [10].

As a result we can say that in this work we obtained better results without using the Ziman formula.

\section{Appendix}

In this appendix first we evaluate $\alpha_{r}^{\prime}$ from formula (3.2)

$$
\begin{aligned}
\alpha_{r}^{\prime}= & \frac{2 \pi}{3 \hbar^{3}} \sum_{k} n_{k}^{\prime} \frac{V}{4 \pi^{2}} \int_{0}^{\infty} \mathrm{d}(\hbar q) \hbar^{4} q^{4}|S(q)|^{2} \vartheta^{2}(q) \\
& \times \int_{-1}^{0} \mathrm{~d} \nu \delta^{\prime}\left(\frac{\hbar^{2} k q}{m} \nu+\frac{\hbar^{2} q^{2}}{2 m}\right),
\end{aligned}
$$

where $\mathrm{d} \phi$ integration has been carried out. If we use the relation

$$
\delta^{\prime}[f(x)]=\frac{1}{\left|f^{\prime}\left(x_{0}\right)\right| f^{\prime}\left(x_{0}\right)}\left[\delta^{\prime}\left(x-x_{0}\right)+\frac{f^{\prime \prime}\left(x_{0}\right)}{f^{\prime}\left(x_{0}\right)} \delta\left(x-x_{0}\right)\right],
$$

with $x_{0}$ being the root of the $f\left(x_{0}\right)=0$, we have

$$
\delta^{\prime}\left(\frac{\hbar^{2} k q}{m} \nu+\frac{\hbar^{2} q^{2}}{2 m}\right)=\frac{m^{2}}{\hbar^{2} k^{2} \hbar^{2} q^{2}} \delta^{\prime}\left(\nu+\frac{\hbar q}{2 \hbar k}\right) .
$$

The root $\nu_{0}$ is given by $\nu_{0}=-\hbar q / 2 \hbar k$. If we substitute (A.3) into (A.1) and do necessary integrations 


$$
\begin{aligned}
\alpha_{r}^{\prime}= & \frac{2 \pi}{3 \hbar^{3}} \frac{V m^{2}}{4 \pi^{2}} \sum_{k} \frac{n_{k}^{\prime}}{\hbar^{2} k^{2}} \int_{0}^{\infty} \mathrm{d}(\hbar q) \hbar^{2} q^{2}|S(q)|^{2} \vartheta^{2}(q) \\
& \times\left[\delta\left(\frac{\hbar q}{2 \hbar k}\right)-\delta\left(-1+\frac{\hbar q}{2 \hbar k}\right)\right] .
\end{aligned}
$$

No contribution comes from the first delta function inside the square bracket to $\alpha_{r}^{\prime}$, but when we calculate $\alpha_{r}^{\prime \prime}$ later on, the corresponding term then will be $\hbar \delta^{\prime}(h q / 2 \hbar k)$ and there will be contribution owing to the relation $x \delta^{\prime}(x)=-\delta(x)$. After turning the $\hbar k$ sum into integration, the integral over $\hbar k$ should be made dimensionless in order to carry out it numerically, so we define $Q=\hbar q / \hbar k_{\mathrm{f}}$, $E_{\mathrm{f}}=\hbar^{2} k_{\mathrm{f}}^{2} / 2 m$. By remembering the relation between the structure amplitude and the structure factor

$$
|S(Q)|^{2}=\frac{\Omega}{V} a(Q)
$$

we can write $\alpha_{r}^{\prime}$ in the form

$$
\alpha_{r}^{\prime}=\frac{V \beta m^{2} k_{\mathrm{f}}^{4} \Omega}{24 \pi^{3} \hbar^{2}} I_{1},
$$

where

$$
I_{1}=\int_{0}^{\infty} \mathrm{d} Q Q^{3} a(Q) \vartheta^{2}(Q) \frac{\exp \left[\beta E_{\mathrm{f}}\left(\frac{Q^{2}}{4}-1\right)\right]}{\left\{\exp \left[\beta E_{\mathrm{f}}\left(\frac{Q^{2}}{4}-1\right)\right]+1\right\}^{2}}
$$

To carry out this integral numerically we need $a(Q)$ and $\vartheta(Q)$ values which were read from Figs. 1 to 6.

For $\alpha_{r}^{\prime \prime}$ after carrying out the necessary integrations in the same way we obtain

$$
\alpha_{r}^{\prime \prime}=\frac{V \beta^{2} m^{2} k_{\mathrm{f}}^{4} \Omega}{24 \pi^{3} \hbar} I_{2}
$$

where

$$
\begin{aligned}
I_{2}= & \frac{1}{\beta E_{\mathrm{f}}} \int_{0}^{\infty} \mathrm{d} Q Q a(Q) \vartheta^{2}(Q) \frac{\exp \left[\beta E_{\mathrm{f}}\left(\frac{Q^{2}}{4}-1\right)\right]}{\left\{\exp \left[\beta E_{\mathrm{f}}\left(\frac{Q^{2}}{4}-1\right)\right]+1\right\}^{2}} \\
& +\frac{1}{\beta E_{\mathrm{f}}} \int_{0}^{\infty} \mathrm{d} Q Q^{2} a^{\prime}(Q) \vartheta^{2}(Q) \frac{\exp \left[\beta E_{\mathrm{f}}\left(\frac{Q^{2}}{4}-1\right)\right]}{\left\{\exp \left[\beta E_{\mathrm{f}}\left(\frac{Q^{2}}{4}-1\right)\right]+1\right\}^{2}}
\end{aligned}
$$

During the evaluation of $I_{2}$ integral a term involving $\mathrm{d}(a(Q)) / \mathrm{d} Q=a^{\prime}(Q)$ also appears,

$$
\frac{1}{\beta E_{\mathrm{f}}} \int_{0}^{\infty} \mathrm{d} Q Q^{2} a^{\prime}(Q) \vartheta^{2}(Q) \frac{\exp \left[\beta E_{\mathrm{f}}\left(\frac{Q^{2}}{4}-1\right)\right]}{\left\{\exp \left[\beta E_{\mathrm{f}}\left(\frac{Q^{2}}{4}-1\right)\right]+1\right\}^{2}}
$$


but we can show that this term vanishes for the homogeneous case. To see this we use the definition of structure factor

$$
a(Q)=\frac{1}{N^{2}} \sum_{i, j} \exp \left[-j \boldsymbol{Q}\left(\boldsymbol{R}_{i}-\boldsymbol{R}_{j}\right)\right]
$$

in Eq. (A.10) with $\boldsymbol{R}_{i}, \boldsymbol{R}_{j}$ showing the atomic site positions in the sample which are considered to be irregular. When derivative of $a(Q)$ is taken, a factor $\left(\boldsymbol{R}_{i}-\boldsymbol{R}_{j}\right)$ comes in front of the exponential factor, afterward we take the configuration averages of both $\alpha_{r}^{\prime}, \alpha_{r}^{\prime \prime}$ and this makes the term in Eq. (A.10) vanish. In Ref. [2] this term was present in the expression of $I_{2}$ integral. However the inclusion or the omission of this term does not change the results very much. In that work structure factor curve given by others were used as input data. Whereas here in this work we are able to draw the structure factor curves ourselves as described in the text. In this work it has been possible to use more accurate input data and accordingly we obtained better results.

\section{References}

[1] J.M. Ziman, Philos. Mag. 6, 1013 (1961).

[2] B. Ünal, B. Alkan, J. Phys. Soc. Jpn. 62, 2425 (1993).

[3] N.W. Ashcroft, J. Lekner, Phys. Rev. 145, 83 (1966).

[4] N.W. Ashcroft, D.C. Langreth, Phys. Rev. 159, 500 (1967).

[5] J.-G. Gassert, M. Mayoufi, M.-C. Bellissent-Funel, J. Phys., Condens. Matter 1, 2409 (1989).

[6] S. Wang, S.K. Lai, C.B. So, J. Phys. F, Metal Phys. 10, 445 (1980).

[7] S. Wang, S.K. Lai, J. Phys. F, Metal Phys. 10, 2717 (1980).

[8] F. Kirchhoff, J.M. Holender, M.J. Gillan, Phys. Rev. B 54, 190 (1996).

[9] P.L. Silvestrelli, A. Alavi, M. Parrinello, Phys. Rev. B 55, 15515 (1997).

[10] R. Kaschner, M. Schöne, G. Seifert, G. Pastore, J. Phys., Condens. Matter 8, L653 (1996).

[11] V. Heine, M.L. Cohen, D. Weaire, Solid State Phys., Academic Press, New York 1970 .

[12] W.A. Harrison, Pseudopotentials in the Theory of Metals, Benjamin, New York 1966.

[13] T.E. Faber, J.M. Ziman, Philos. Mag. 11, 153 (1965).

[14] N.W. Ashcroft, D.C. Langreth, Phys. Rev. 156, 685 (1967).

[15] U. Werner, R. Fresard, Z. Phys. B 104, 379 (1997).

[16] B. Ünal, T. Altanhan, B. Alkan, Prog. Theor. Phys. 88, 485 (1992).

[17] M. Hasegawa, Solid State Commun. 11, 531 (1972).

[18] Y. Waseda, The Structure of Non-Crystalline Materials, McGraw-Hill, New York 1980.

[19] J. Hannephof, W. Van Der Lugt, G.W. Wright, T. Meriën, Physica 61, 146 (1972). 